\title{
Notwendigkeit einer Hämoglobin-Bestimmung nach Knie-TEP
}

Knie-Totalendoprothetik kann einen erheblichen Blutverlust zur Folge haben und zu einem symptomatischen Abfall des HbWertes (Hb: Hämoglobin) führen. Die vorliegende Studie untersucht mithilfe eines Kollektivs von Knie-TEP-Patienten (TEP: totale Endoprothese) die Notwendigkeit postoperativer Routine-Hb-Bestimmungen anhand präoperativer Faktoren, wenn diesen intraoperativ intraartikulär Tranexamsäure (TXA) appliziert wurde.

Goyal N, Kaul R, Harris IA et al. Is there a need for routine postoperative hemoglobin level estimation in total knee arthroplasty with tranexamic acid use? Knee 2016; 23 : 310-313

Goyal et al. konnten anhand Ihrer Daten zeigen, dass der präoperative Hb-Wert der einzig signifikante Prädiktor postoperativer Bluttransfusionen ist $(\mathrm{p}<0,0001)$. Geschlecht, Alter, Größe, Gewicht und BodyMass-Index (BMI) hatten keinen Einfluss auf das postoperative Transfusionsrisiko.

\section{Methodik}

$\nabla$

In die vorliegende retrospektive Studie wurden Patienten nach primärer KnieTEP-Implantation mit intraoperativ intraartikulär applizierter TXA eingeschlossen.

Alle Patienten wurden über einen medialen parapatellaren Zugang operiert und erhielten zementierte PS-Implantate (PS: posterior stabilisiert) mit Retropatellarersatz.

Nach dem Wundverschluss erhielten alle Patienten $3 \mathrm{~g}$ TXA über einen intraartikulären Schmerzkatheter, der stets am 1. postoperativen Tag entfernt wurde. Wunddrainagen wurden nicht verwendet. Die Hb-Bestimmungen erfolgten präoperativ sowie am 1 . und 2 . postoperativen Tag.

Erforderliche Transfusionen wurden nach den Leitlinien der Australian National Blood Authority gegeben. (Generell bei einem $\mathrm{Hb}<7 \mathrm{~g} / \mathrm{dl}$ bzw. bei Begleiterkrankungen wie dem akuten Myokardinfarkt oder der zerebrovaskulären Ischämie auch bei Hb-Werten zwischen $7-10 \mathrm{~g} / \mathrm{dl}$.)

Die präoperative Datensammlung ( $\mathrm{Hb}$, Geschlecht, Alter, Größe, Gewicht und BMI) wurde im Hinblick auf das postoperative Transfusionsrisiko analysiert, wobei $\mathrm{p}<0,05$ als signifikant galt.

\section{Ergebnisse}

487 Patienten wurden untersucht (292 Frauen, 195 Männer; Durchschnittsalter 68,1 Jahre; mittlere Größe 1,67 m; mittleres Gewicht $84,8 \mathrm{~kg}$ ).

Bluttransfusionen waren bei 12 (2,5\%) der 487 Patienten erforderlich. Einzig der präoperative $\mathrm{Hb}$-Wert stellte sich hierbei als signifikanter Prädiktor heraus. Keiner der 204 Patienten mit einem präoperativen $\mathrm{Hb}>14,0 \mathrm{~g} / \mathrm{dl}$ erhielt eine Blutübertragung, wohingegen $4,2 \%$ der Patienten mit einem präoperativen $\mathrm{Hb}<14,0 \mathrm{~g} / \mathrm{dl}$ trans-

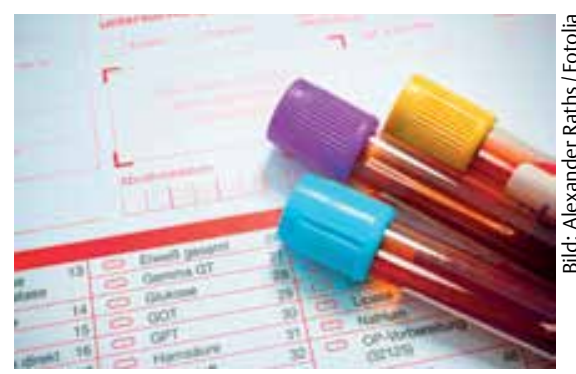

fundiert wurden. Der mittlere präoperative Hb-Wert bei Patienten, die transfundiert wurden, lag bei $11,53 \mathrm{~g} / \mathrm{dl}$, verglichen mit $13,81 \mathrm{~g} / \mathrm{dl}$ bei Patienten, die keine Transfusion erhalten mussten. Geschlecht, Alter, Größe, Gewicht und BMI hatten keinen Einfluss auf das postoperative Transfusionsrisiko.

Die Risikoberechnung ergab, dass eine Reduktion des präoperativen Hb-Wertes von $0,1 \mathrm{~g} / \mathrm{dl}$ die Chancen einer Transfusion um ca. $11 \%$ (Odds Ratio $=0,88,95 \%$ Konfidenzintervall =0,84-0,93) erhöht.

\section{Fazit \\ $\nabla$}

Postoperative Hb-Bestimmungen sind eine anerkannte Routine in der Knieendoprothetik, da Blutungen trotz moderner OP-Techniken ein Problem darstellen.

Verschiedene Studien haben Prädiktoren für eine postoperative Bluttransfusion mit heterogenen Ergebnissen untersucht. Die vorliegende Studie ist nach Kenntnis des Autors jedoch die erste, die diese im Rahmen der Knieendoprothetik unter Verwendung von TXA betrachtet.

TXA reduziert als Antifibrinolytikum Blutungen. Alshryda et al. konnten bereits nachweisen, dass die intraartikuläre TXAApplikation bei Knie-TEP-Patienten das absolute Risiko einer Bluttransfusion von $16,7 \%$ auf $1,3 \%$ reduziert. Eine Meta-Analyse desselben Autors zeigte, dass TXA den Blutverlust im Mittel um $591 \mathrm{ml}(\mathrm{p}<0,001)$ reduziert.

Die vorliegende Studie zeigt nun die Bedeutung des präoperativen $\mathrm{Hb}$ als starken Prädiktor für das postoperative Transfusionsrisiko, wobei das retrospektive Studiendesign jedoch eine Einschränkung darstellt.

Basierend auf den Ergebnissen rät der Autor bei Knie-TEP-Patienten mit intraartikulärer TXA-Anwendung und einem präoperativen $\mathrm{Hb}>14,0 \mathrm{~g} / \mathrm{dl}$ von postoperativen Hb-Routineuntersuchungen ab. Klinische Symptome und atypische postoperative Ereignisse sollten jedoch eine schnelle $\mathrm{Hb}$-Kontrolle nach sich ziehen.

\section{Peter Paes}

Orthopädische Klinik der Medizinischen

Hochschule Hannover

petersebastian.paes@diakovere.de 